\title{
Editorial
}

\section{Who needs ventricular stimulation studies?}

Two randomised studies reported in the New England Fournal of Medicine have cast doubt on the value of ventricular stimulation studies in the assessment of antiarrhythmic drug therapy in patients with symptomatic sustained ventricular arrhythmias. In the study by Steinbeck and coworkers 115 patients with inducible ventricular arrhythmias were assigned to treatment with electrophysiologically guided drug therapy or with empirical metoprolol. ${ }^{1}$ During a mean follow up of 23 months the incidence of recurrent arrhythmia and sudden death was almost identical in the two groups. Steinbeck et al concluded that electrophysiologically guided drug therapy did not improve the overall outcome of patients with sustained ventricular arrhythmias. In the Electrophysiologic Study Versus Electrocardiographic Monitoring (ESVEM) study 486 patients with both inducible ventricular arrhythmias and frequent ventricular extrasystoles during Holter monitoring were randomly assigned to antiarrhythmic therapy guided by either electrophysiological study or by Holter monitoring. ${ }^{23}$ Over a 6 year follow up there was no significant difference between the two study groups in terms of recurrence of arrhythmia, sudden death, or total mortality. The ESVEM group recommended that treatment with sotalol (the most effective drug in the trial) and assessment of its potential efficacy by Holter monitoring was a reasonable initial strategy in patients with characteristics similar to those of their study population.

A casual reader of the New England Fournal of Medicine might be forgiven for thinking that there are now few, if any, indications for ventricular stimulation studies in the management of patients with symptomatic sustained ventricular arrhythmias.

\section{Current practice}

Ventricular stimulation studies are widely used for the management of recurrent sustained ventricular tachycardia and cardiac arrest in both Europe and the United States. This widespread use is not, however, based on an assumption that electrophysiologically guided therapy itself improves outcome in these patients. ${ }^{4}$ Indeed it has been frequently stated that this may not be so, and the results of CAST in 1989 underlined this possibility. ${ }^{5}$ It is increasingly recognised that antiarrhythmic drug therapy, particularly with agents of Vaughan Williams class 1 , may have a deleterious effect on overall mortality in several different groups of patients. ${ }^{6}$

Programmed electrical stimulation of the ventricle is performed principally as a means of assessing prognosis in patients with recurrent ventricular arrhythmias, rather than as a means of selecting a particular antiarrhythmic drug. Gone are the days of prolonged hospital admissions with repeated testing of different classes of antiarrhythmic drugs or different drugs from within the same class. More relevant is the ability of the technique to provide prognostic information that, in conjunction with the clin- ical history (ventricular tachycardia or cardiac arrest) and an index of ventricular function, allows rational selection of those patients most likely to benefit from non-pharmacological therapy. For instance, it has been realised for some time that the prognosis of patients with ventricular arrhythmias that are not inducible by ventricular stimulation is relatively good. ${ }^{7}$ This is borne out by the study of Steinbeck et $a l,{ }^{1}$ in which recurrent arrhythmia and sudden death were significantly less common in patients with non-inducible arrhythmia at baseline than in patients with inducible arrhythmia, irrespective of whether the patients with inducible arrhythmia were assigned to electrophysiologically guided drug therapy or to empirical metoprolol.

Similarly patients with inducible arrhythmias that become non-inducible on drug therapy are known to have a better prognosis than those in whom the arrhythmia remains inducible despite drug therapy. ${ }^{78}$ This has been confirmed by several studies with different drugs including that of Steinbeck and coworkers. Persistence of arrhythmia inducibility despite drug therapy has been shown to be an independent predictor of sudden death or recurrent cardiac arrest. ${ }^{17}$ This is not to suggest, of course, that the drugs themselves are actually improving prognosis: drug suppression may be merely a marker of a prognosis that is already good.

\section{Era of the transvenous implantable defibrillator}

Assessment of prognosis in patients with sustained ventricular arrhythmias has become critically important since the advent of cardioverter defibrillators that can be implanted transvenously without thoracotomy. Despite the conspicuous absence of a randomised controlled trial showing prolonged overall survival with the use of implantable cardioverter defibrillators in high risk patients, even the critics of the devices have conceded that they are effective in preventing sudden death from cardiac tachyarrhythmias. ${ }^{9}$ Transvenous cardioverter defibrillators can now be implanted with an operative mortality less than $0.5 \%$ and a 1 year mortality from sudden death of less than $1 \%$, suggesting the likelihood of a higher overall survival than with previous epicardial patch systems. ${ }^{10}$ These results, in conjunction with the relative inefficacy of most antiarrhythmic drugs, the currently limited role of ablation techniques, and relatively high mortality associated with surgery for ventricular tachycardia, have caused a major shift to device therapy in many centres. It is becoming increasingly clear that patient groups other than those resuscitated from ventricular fibrillation are likely to benefit from these devices. Widespread implantation is now a practical option and cost restraints have come to the fore. Selection of patients for device therapy is increasingly based on costbenefit issues, with those at highest risk from sudden death having the most to gain.

A large body of information is available on the 
prognostic value of ventricular stimulation studies $^{711-13}$ and together with the clinical history and assessment of ventricular function, such studies are an integral part of the assessment of risk in patients with sustained ventricular arrhythmias. In many centres the decision to implant a device is based on the inducibility of a ventricular arrhythmia at baseline or in the presence of a single antiarrhythmic drug. ${ }^{14}$ Usually the response to a class 1 agent (often intravenous procainamide) is used for this purpose but there is evidence that other drugs, including amiodarone, may be as useful in such tests. The likelihood of tachycardia induction is higher after amiodarone (approximately $80 \%$ of patients) than after administration of class 1 agents and the effect of amiodarone on the rate of the induced arrhythmia provides the principal means of prognostic assessment. ${ }^{12} 15$

Although the ESVEM study showed that there was no difference in terms of arrhythmia recurrence between drug therapy selected by invasive and non-invasive techniques (they seemed equally ineffective) the study was not designed to compare the prognostic value of the two techniques in terms of mortality and did not have the power to do so. There is considerably less information on the prognostic power of Holter monitoring in the setting of recurrent sustained ventricular arrhythmias than on that of programmed electrical stimulation. Newer noninvasive means of risk assessment (heart rate variability, signal averaged electrocardiography) have shown promise in patients assessed after infarction ${ }^{16}$ but have yet to be fully evaluated in patients with recurrent ventricular arrhythmias. Consequently the suggestion that Holter assessment of drug therapy is a reasonable initial strategy in patients with sustained ventricular arrhythmias cannot be supported.

In summary, it is clear that the issues concerning management of sustained ventricular arrhythmias have changed since the design of the ESVEM study in the middle 1980s. There has been a dramatic trend away from antiarrhythmic drugs and towards device therapy. The priority now is to be able to stratify patients with ventricular arrhythmias prognostically so as to determine those likely to benefit most from a limited resource. At present programmed electrical stimulation of the ventricle together with evaluation of the clinical history and assessment of ventricular function is the best way to do this.

Academic Department of Cardiology,

CLIFFORD J GARRATT

Glenfield Hospital,

Groby Road,

Leicester LE3 9QP

1 Steinbeck G, Andresen D, Bach P, et al. A comparison of electrophysiologically guided antiarrhythmic drug therapy with beta-blocker therapy in patients with symptomatic sustained ventricular tachyarrhythmias. $N$ Engl f Med 1992;327:987-92.

2 Mason JW. A comparison of electrophysiologic testing with Holter monitoring to predict antiarrhythmic drug efficacy for ventricular tachtoring to predict antiarrhythmic drug efficacy

3 Mason JW. A comparison of seven antiarthythmic drugs in patients with ventricular tachyarrhythmias. N Engl ₹ Med 1993;329:452-8.

4 Fromer M, Shenasa M. A critical reapraisal of serial electrophysiologic drug testing for sustained ventricular tachycardia. Am Heart $f 1987$; 114:1537-43.

5 The Cardiac Arrhythmia Suppression Trial (CAST) investigators. Preliminary report: effect of encainide and flecainide on mortality in a randomised trial of arrhythmia suppression after myocardial infarction. N Engl F Med 1989;321:406-12.

6 Coplen SE, Antman EM, Berlin JA, et al. Efficacy and safety of quinidine therapy for maintenance of sinus rhythm after cardioversion: a metaanalysis of randomised controlled trials. Circulation 1990;82:1106-16.

7 Wilber DJ, Garan H, Finkelstein D, et al. Out-of-hospital cardiac arrest: use of electrophysiologic testing in the prediction of long-term outcome. N Engl F Med 1988;318:19-24.

8 Ruskin JN, DiMarco JP, Garan H. Out-of-hospital cardiac arrest. Electrophysiological observations and selection of long term antiarrhythmic therapy. $N$ Engl $₹$ Med 1980;303:607-13.

9 Fogoros RN. The effect of the implantable cardioverter defibrillator on sudden death and on total survival. PACE 1993;16:506-10.

10 Saksena S, Camm J. Implantable defibrillators for prevention of sudden death: technology at a medical and economic crossroad. Circulation 1992;85:2316-21.

11 Swerdlow CD, Winkle RA, Mason JW. Determinants of survival in patients with ventricular tachyarrhythmias. $N$ Engl $f$ Med 1983;308: patients $1436-42$.

12 Waller TJ, Kay HR, Speilman SR, et al. Reduction in sudden death and total mortality by antiarrhythmic therapy evaluated by electrophysiologic drug testing: criteria of efficacy in patients with sustained ventricular tachyarrhythmia. F Am Coll Cardiol 1987;10:83-9.

13 Powell AC, Fuchs T, Finkelstein DM, et al. Influence of implantable cardioverter-defibrillator on the long-term prognosis of survivors of out-ofhospital cardiac arrest. Circulation 1993;88:1083-92.

14 Kuchar DL, Rottman J, Berger E, et al. Prediction of successful suppression of sustained ventricular tachyarrhythmias by serial drug testing
from data derived at the initial electrophysiologic study. $\mathcal{F} \mathrm{Am}$ Coll Cardiol 1988;12:982-8.

15 Kadish AH, Buxton AE, Waxman HL, et al. Usefulness of electrophysiologic study to determine the clinical tolerance of arrhythmia recurrences during amiodarone therapy. $f \mathrm{Am}$ Coll Cardiol 1987;10:90-6.

16 Farrell TG, Bashir Y, Cripps T, et al. Risk stratification for arrhythmic events in post infarction patients based on heart rate variability, ambulatory electrocardiographic variables and the signal averaged electrocardiogram. $₹$ Am Coll Cardiol 1991;18:687-97. 